\title{
MONTE-CARLO SIMULATION OF LIGHT TRANSPORT FOR NIRS MEASUREMENTS IN TUMORS OF ELLIPTIC GEOMETRY
}

\author{
Mojca Pavlin*, Tomaž Jarm \& Damijan Miklavčič \\ Univ.of Ljubljana, Faculty of Electrical Eng., Trzaska 25, SI-1000 Ljubljana, SLOVENIA \\ *Tel.+386611768264,Fax+386611264658,E-mail:mojca@svarun.fe.uni-lj.si
}

\begin{abstract}
Propagation of light in a highly scattering medium such as biological tissue is difficult to study. For complex geometry and multilayer structures computer simulation has to be used for light transport analysis. A Monte Carlo model of light propagation in tissue has been applied for the purpose of better understanding of the results of near-infrared spectroscopy (NIRS) measurements in experimental tumors. The major objective was to determine the percentage and location of the illuminated area in tumor and to estimate fraction of NIRS signal originating from the underlying tissues. Values of optical parameters used in the model were taken from literature. Tumor shape was approximated with a rotational ellipsoid. Computer simulations were made for two positions of optodes: reflectance and transmittance mode. Results of simulations indicate that in both configurations the majority of signal originates from tumor and not from surrounding tissue. In reflectance mode collected light comes from limited area near the optode whereas in transmittance mode the collected light illuminate almost whole tumor. This difference between the two modes is valid for all tissue parameters.
\end{abstract}

Key words: Near-infrared spectroscopy, Monte-Carlo model, light transport, tumor

\section{INTRODUCTION}

Propagation of light in tissue is a complex phenomenon due to strong scattering, insufficiently known optical parameters and heterogeneous structure of tissue. Even in simple geometry it is hard to solve this problem theoretically and impossible for more complex geometry. For this reason

Oxygen Transport to Tissue XXIV, edited by

Dunn and Swartz, Kluwer Açademic/Plenum Publishers, 2003 
different numerical approaches are used, where one of most commonly used is Monte-Carlo method: Understanding and quantitative analysis of light transport in tissue is important for medical applications of different optical methods. One of the recently developed optical methods is near--infrared spectroscopy (NIRS), which enables noninvasive measurement of oxygenation and perfusion in soft tissue in vivo. NIRS method uses a difference in the light absorption spectra of oxyhemoglobin and deoxihemoglobin in near-infrared spectrum to measure concentrations of oxy- and deoxyhemoglobin. From relative change of light attenuation concentration changes of the absorbers can be determined.

The major problem related to quantification of NIRS data arises from the fact that light attenuation in tissue originates not just from absorption but also from light scattering. Light loss due to scattering depends on tissue parameters and measurement geometry and results in a non-linear relationship between absorption changes and attenuation changes. One of the problems of experiments with NIRS method is also nonhomogeneous structure of tissue. As a result of tissue inhomogenity signals may actually be obtained from different tissue types, in different physiological states [1]. Another complication follows from the fact that due to strong scattering of light in tissue the pathlength is greater than the distance $d(\mathrm{~cm})$ between emitting and receiving optode. The traveled average distance $d_{o p}(\mathrm{~cm})$ optical path is larger by a differential pathlength factor $B$, defined as: $B=d_{o p t} /$ $d$. It was proven for the attenuation of light of certain wavelength in tissue that it agrees with modified Beer Lamber's law [1]:

$$
A=\log \left(J / J_{0}\right)=\sum_{\mathrm{i}}=\alpha_{i} c_{i} B d+G
$$

where $J_{0}\left(\mathrm{~W} / \mathrm{m}^{2}\right)$, and $J\left(\mathrm{~W} / \mathrm{m}^{2}\right)$ represent intensity of inward and outward light flux, $A(\mathrm{OD})$ is attenuation, $B$ is differential pathlength factor, $\alpha\left(\mu \mathrm{M}^{-}\right.$ $\left.{ }^{1} \mathrm{~cm}^{-1}\right)$ specific extinction coefficient, $c\left(\mu \mathrm{M}^{-1}\right)$ is concentration of the absorber and index $i$ stands for different absorbers. Parameter $G$ represents unknown light losses due to scattering, undefined absorption and other undefined losses.

Our work was related to measurements of oxygenation with NIRS method on small subcutaneous tumors [2], experimental configuration of these measurements is presented in figure 1a. The main problem in this kind of measurements is unknown fraction of light originating from tumor itself due to its small size and position of the optodes. Therefore our main objective was to estimate how much signal emerges from underlying tissues and to determine the illuminated area of tumor tissue for reflectance and transmittance modes. 

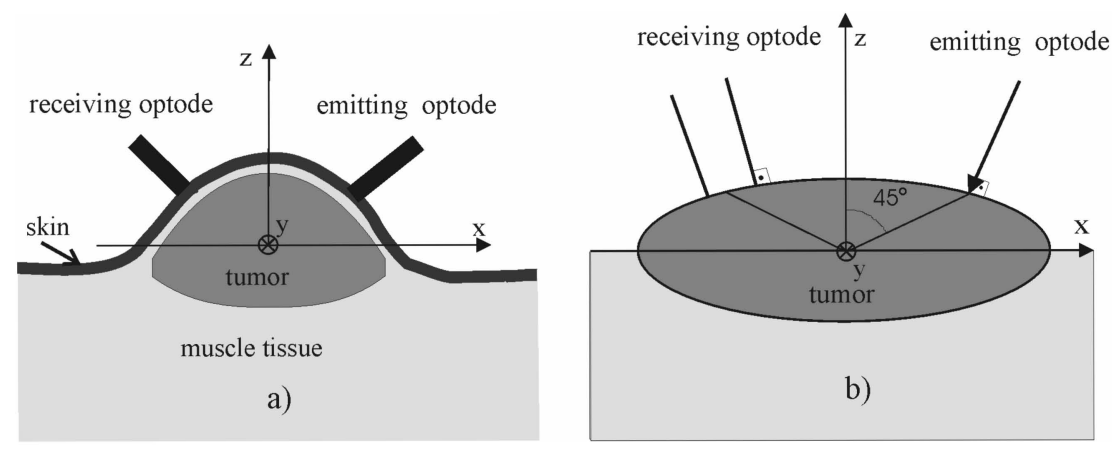

Figure 1. Position of optical fibers-optodes in transmittion mode a) for NIRS measurements in tumor b) in simulations.

A Monte-Carlo model of light propagation in tissue has been applied. Values of optical parameters for near-infrared wavelengths have been selected in the range of values reported in literature. We have also studied how changing the values of parameters affects attenuation and differential pathlength factor of photons.

\section{METHODS}

Monte-Carlo method has been widely applied in radiation transport studies. It is a stochastic method that simulates photon propagation as a random walk from one interaction to another. Transport of light in the tissue can be described by computer simulation of appropriately weighted random absorption and scattering interactions [3,4,5]. A three-dimensional MonteCarlo model based on transport equation [5] was developed in our study. Parameters for the model are the following;

- Parameter $g$ - anisotropy factor which is defined as the average scattering angle $g=<\cos \theta>$, where larger $\mathrm{g}$ means more scattering in the forward direction. For scattering in tissue it was shown that it is mainly forward scattering and can be approximated with Henyey-Greenstein function.

- Total attenuation coefficient $\mu_{t}\left(\mu_{t}=\mu_{a}+\mu_{s}\right)$; the sum of scattering coefficient $\mu_{\mathrm{s}}$ and absorption coefficient $\mu_{\mathrm{a}}$.

- Albedo, defined as: $w=\mu_{s} /\left(\mu_{a}+\mu_{s}\right)$.

- Values of optical parameters used in the model were taken from literature [6] and were for albedo $(w)$ in range from 0.994 to 0.998 , for scattering coefficient $\left(\mu_{\mathrm{s}}\right)$ from 160 to $410 \mathrm{~cm}^{-1}$ and for mean cosine of the scattering angle $(g)$ from 0.93 to 0.97 .

Tumor shape was approximated with rotational ellipsoid with orthogonal diameters being $a=8 \mathrm{~mm}, b=8 \mathrm{~mm}$ and $\mathrm{c}=3 \mathrm{~mm}$ long (volume $=96 \mathrm{~mm}^{3}$ ). In 
transmittance mode two optodes were placed symmetrically at angle 45 degrees to the rotational axis (Figure 1b). In reflectance mode both optodes were placed on the same spot at angle 45 degrees to the rotational axis. The emitting optode was approximated by a point source in both configurations, whereas the receiving optode had finite dimensions of the one used in measurements. Fiber diameter was $1586 \mu \mathrm{m}$ and numerical aperture was 0.55 . The basic simulation steps were:

1. Point source photon generation of a normally incident beam.

2. At each interaction point scattering or absorption was chosen randomly, proportionally to the probability of each.

3. Pathway generation. The distance between two successive interactions was calculated as: $l=1 / \mu_{t}(-\ln R N)$, where $R N$ is a random number from a sequence of independent uniformly distributed random numbers. With this formula we generated an exponential distribution of interaction path lengths with mean free path $s=1 / \mu_{t}$.

4. New direction was randomly selected from appropriate distribution function. The azimuth angle $\Phi$ and the cosine of the scattering angle $\theta$ with respect to the previous direction were generated by:

$$
\begin{gathered}
\Phi=2 \pi R N, \\
\cos \theta=\left[\left(1+g^{2}\right)-\left(1-g^{2}\right)^{2}-(1-g+2 g R N)^{-2}\right][2 g]^{-1},
\end{gathered}
$$

where Eq. (4) is derived from the Henyey-Greenstein phase function.

5. At each point we checked whether the photon crossed the fiber tip at an angle smaller than numerical aperture. If so, all scattering points for this photon were stored. Typically 100,000 photons were generated to detect 200 photons in transmittance mode and 1000 photons in reflectance mode.

We used Fresnel's laws to calculate the possibility of reflection and refraction for each collision of a photon with an interface between air and tissue [7]. Reflections at tissue-fiber surfaces were ignored due to small difference in refractive index. The tissue volume was defined with the sum of ellipsoid volume and semi-infinite space defined by $\mathrm{z}<0$.

\section{RESULTS}

All scattering points of detected photons were stored. To present light distribution graphically we took two projections of all scattering points in $x z$ 
and $y z$ plane. We separated the planes into array of fields $55 \mu \mathrm{m} \times 55 \mu \mathrm{m}$ large and counted the number of appropriately weighted interactions in each field.

Figures 2 and 3 represent light distribution drawn in planes $x z$ and $y z$ of simulation with parameters being $w=0.998, g=0.96$ and $s=25 \mu \mathrm{m}$.

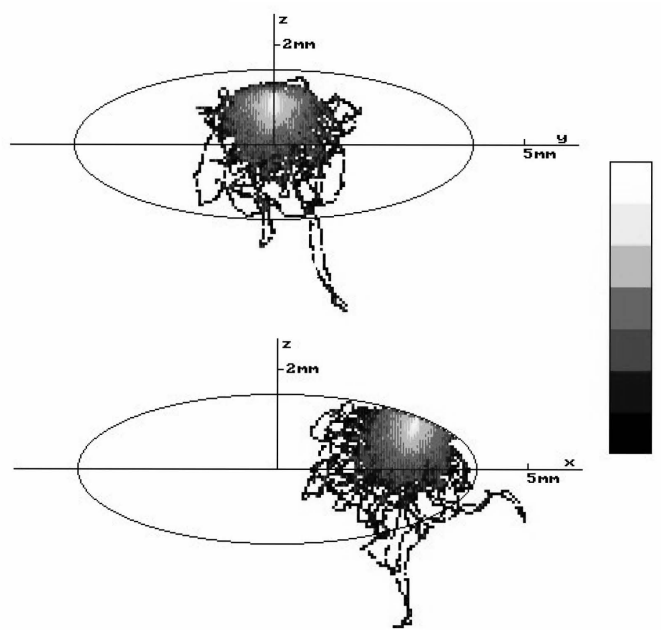

Figure 2. Light intensity distribution for reflectance mode in a) $x z$ plane and b) $y z$ plane. Gray scale is logarithmic and relative to maximum of intensity, brighter shading represents higher intensity. Parameters of simulation are $g=0.96, w=0.998$ and average path $\mu_{s}^{-1}=s=25 \mu \mathrm{m}$.

Density of interactions represents measure of light intensity at specific place and is represented in a gray scale. The scale is logarithmic and relative to the maximum of intensity, where brighter shading represent higher intensity.

We can see the difference in illuminated volume between the two modes. In the reflectance mode the collected light originates from a limited area (approx. $5 \mathrm{~mm}^{3}$ ) near the optode. In contrast in the transmittance mode the collected light illuminates approximately $65 \mathrm{~mm}^{3}$ of ellipsoid. It can also be seen from figure 3 that in the transmittance mode a fraction of photons travels outside ellipsoid. 


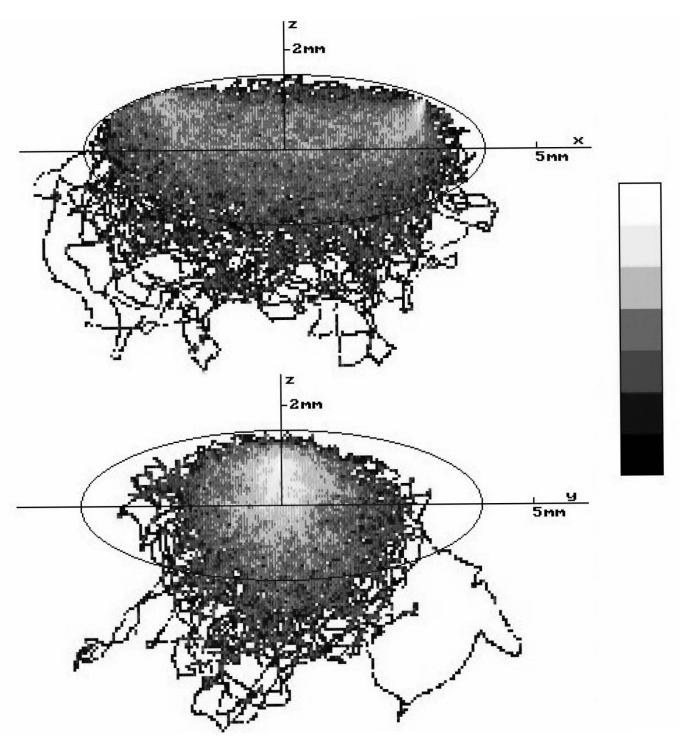

Figure 3. Light intensity distribution for trasmittance mode in a) $x z$ plane and b) $y z$ plane. Gray scale is logarithmic and relative to maximum of intensity, brighter shading represent higher intensity. Parameters of simulations are $g=0.96, w=0.998$ and average path $\mu_{t}^{-}$ ${ }^{\prime}=s=25 \mu \mathrm{m}$.

Table 1. Percentage of the signal originating outside the ellipsoid (1) and fraction of illuminated volume (2) for reflectance and transmittance mode.

\begin{tabular}{|c|c|c|c|c|c|c|c|c|c|}
\hline \multirow{3}{*}{\multicolumn{2}{|c|}{$\%$}} & \multirow{2}{*}{\multicolumn{6}{|c|}{$\frac{\mathrm{g}=0.94[\mathrm{~g}=0.95 \mid \mathrm{g}=0.96] \mathrm{g}=0.97}{\mathrm{~s}=60 \mathrm{um}}$}} & \multicolumn{2}{|c|}{$\mathrm{g}=0.97$} \\
\hline & & & & & & & & $\mathrm{s}=25 \mu \mathrm{m}$ & $\mathrm{s}=35 \mu \mathrm{m}$ \\
\hline & & \multicolumn{6}{|c|}{$w=0.994$} & \multicolumn{2}{|c|}{$w=0.998$} \\
\hline \multirow[t]{2}{*}{1} & ref. & 1.5 & 1.5 & 3.3 & 5. & & 5.4 & 3.0 & 5.8 \\
\hline & tran & 12.5 & 12.5 & 15.5 & 15 & & 18.7 & 15.8 & 26.3 \\
\hline \multirow[t]{2}{*}{2} & refl. & 5.0 & 5.0 & 5.0 & 4 & & 4.1 & 5.1 & 4.9 \\
\hline & $\operatorname{tran}$ & 60 & 77 & 72 & 5 & & 58 & 56 & 60 \\
\hline & & \multicolumn{2}{|c|}{$g=0.97$} & \multicolumn{6}{|c|}{$\mathrm{g}=0.95$} \\
\hline & \multirow[t]{2}{*}{$\%$} & $\begin{array}{c}s=45 \mu \\
m\end{array}$ & $\begin{array}{c}s=60 \mu \\
m\end{array}$ & \multicolumn{6}{|c|}{$\mathrm{s}=60 \mu \mathrm{m}$} \\
\hline & & \multicolumn{2}{|c|}{$\mathrm{w}=0.998$} & \multicolumn{2}{|c|}{$w=0.995$} & \multicolumn{2}{|c|}{$w=0.996$} & $w=0.997$ & $w=0.998$ \\
\hline \multirow[t]{2}{*}{1} & ref & 9.2 & 12.8 & 4.2 & & & .4 & 5.4 & 6.1 \\
\hline & tran & 22.8 & 36.1 & 20. & & & 1.9 & 23.8 & 26.7 \\
\hline \multirow[t]{2}{*}{2} & ref & 4.3 & 3.6 & 4.8 & & & .9 & 4.5 & 3.7 \\
\hline & $\operatorname{tran}$ & 62 & 60 & 68 & & & 59 & 72 & 67 \\
\hline
\end{tabular}


In table 1 percentages of signal (photon interactions) originating outside the ellipsoid (1) and fraction of illuminated volume (2) for reflectance and transmittance mode for 13 different sets of parameters are given. The results demonstrate that $2-9 \%$ and $15-37 \%$ of detected signal for the reflectance mode and the transmittance mode respectively originates from the tissue outside the tumor.

To estimate the illuminated volume we counted number of fields with at least one interaction in both planes. With this we calculated that in reflectance mode only $4-5 \%$ of ellipsoid volume was illuminated in contrast to transmittance mode where $56-72 \%$ of volume was illuminated.

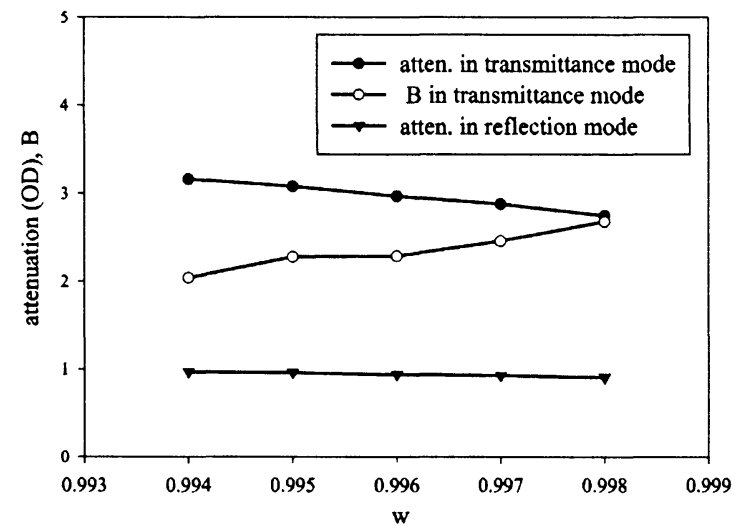

Figure 4. Attenuation (OD) in transmittance and reflectance mode and differential pathlength factor- $B$ in transmittance mode at different values of albedo $w$. Parameters of simulations are $g=0.95$ and $s=60 \mu \mathrm{m}$.

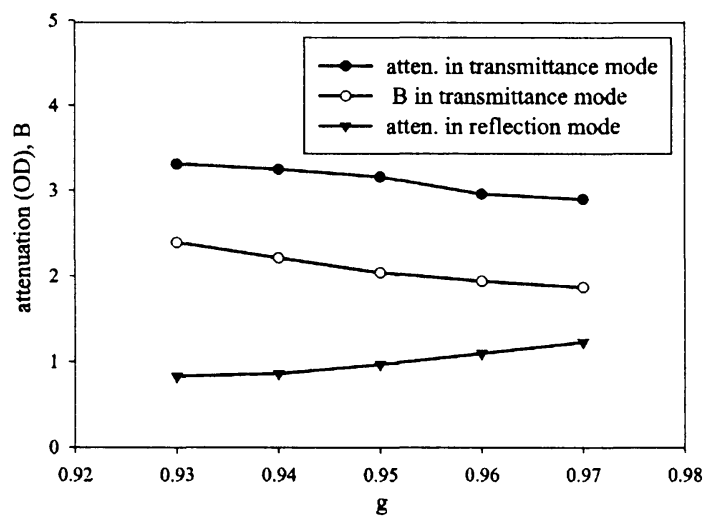

Figure 5. Attenuation (OD) in transmittance and reflectance mode and differential pathlength factor - $B$ in transmittance mode at different values of parameter $g$. Parameters of simulations are $w=0.994$ and $s=60 \mu \mathrm{m}$. 
In figure 5 dependency on anisotropy parameter $g$ is shown. Larger parameter $\mathrm{g}$ means less scattering and more signal in forward and less in backward direction. This results in smaller attenuation and smaller $B$ for transmittance mode and larger attenuation in reflectance mode. In figure 6 dependency of attenuation and parameter $B$ on average free path is shown. If average free path is increased there is less collisions and less light loss, which results in smaller attenuation in transmission mode. Average free path has no significant impact on differential pathlength factor. For all simulations counting errors are $0.07 \mathrm{OD}$ in the transmittance mode and 0.03 OD in the reflectance mode.

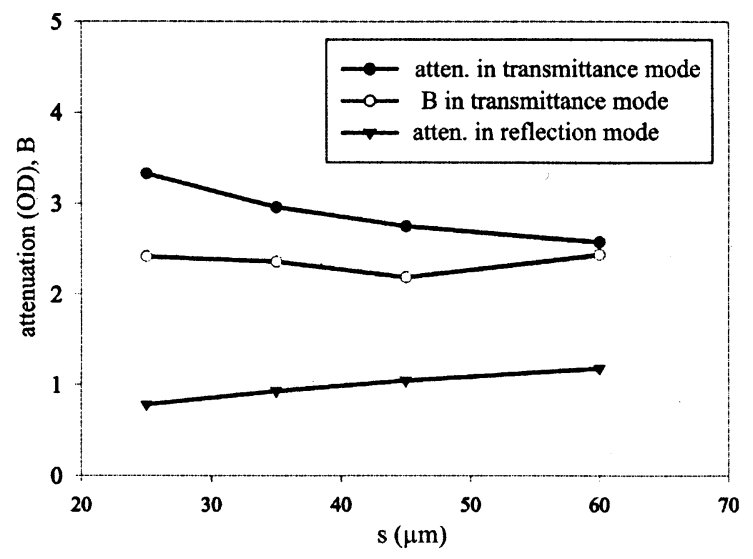

Figure 6. Attenuation (OD) in transmittance and reflectance mode and differential pathlength factor- $B$ in transmittance mode at different values of average path $\mu_{t}^{-1}=s(\mu \mathrm{m})$. Parameters of simulations are $w=0.998$ and $g=0.97$.

\section{DISCUSSION}

Results of simulations indicate that in both measurement configurations the majority of NIRS signal originates from tumor and not from surrounding tissue. In reflectance mode the collected light comes from a limited area (approx. 5\% of volume) near the optode; therefore, we should measure at more different positions to obtain valid results. In contrast to this in the transmittance mode collected light illuminated almost whole tumor (approx. $65 \%$ of the volume) and this is valid for all range of tissue parameters. Although the transmittance mode signal is few orders of magnitude smaller than the reflectance mode signal, the first mode gives more relevant information of overall tumor oxygenation and perfusion. Therefore, for all but smallest tumors the transmittance mode seems to be more appropriate. 
Attenuation and average path of photons do not depend strongly on the values of optical parameters, but much more so on the position of the optodes. The results support our assumptions that most of NIRS signal recorded from small subcutaneous tumors originate from the tumor itself and are not significantly contaminated by the underlying tissues. Since values of parameters vary for different tissue types, we should consider a multilayer structure as a more realistic model.

\section{REFERENCES}

1. Delpy DT, Cope M. Quantification in tissue near-infrared spectroscopy. Phil Trans R Soc Lond B 1997; 649-657.

2. Jarm T, Wickramasinghe YABD, Deakin M, Cemazar M, Miklavcic D, Sersa G, Elder J, Rolfe P, Vodovnik L: Blood Perfusion and Oxygenation of Tumours Following Electrotherapy - A Study by Means of Near Infrared Spectroscopy and Patent Blue Staining. Proceedings of the 9th International Conference on Mechanics in Medicine and Biology, Ljubljana, Slovenia, 1996;339-342

3. Wilson BC, Adam G. A Monte-Carlo model for the absorption and flux distribution of light in tissue. Med Phys 1983;10:824-830.

4. Groenhuis RAJ, Ferwenda HA, Ten Bosch JJ. Scattering and absorption of turbid materials determined from reflection measurments. 1: Theory. Appl Opt 1983;22(16):2456-2462.

5. Ishimaru A. Diffusion of light in turbid media. Appl Opt 1989;28:2210-2215.

6. Beek JF, Blokland P, Posthumus P, Aalders M, Pickering JW, Sterenborg HJCM, van Gemert MJC. In vitro double-integrating-sphere optical properties of tissues between 630 and $1064 \mathrm{~nm}$. Phys Med Biol 1997;42:2255-2261.

7. Bolin FP et al. Refractive index of some mammalian tissues using a fiber optic cladding method. Appl Opt 1989;28(12):24297-2303. 